\section{Journal of Environmental Engineering and Science}

Author Index

Volume 2, 2003

\section{Revue du génie et de la science de l'environment}

Index des auteurs

Volume 2, 2003
Abu-Orf, M., 395

Adnan, A., 315, 473

Adsett, J., 233

Akhand, N., 233

Allen, E.W., S73

Al-Malack, M.H., 155

Aubert, D., 77

Ayala-del-Rio, H.L., 247

Barbeau, B., 237, 281

Bennett, B., 265

Bernier, A.-M., 281

Bhoi, Z.M.P., 307

Bishop, P.L., 27

Blais, J.-F., 1, 9, 355

Bouchard, C., 139

Boutin, C., 383

Bowman, D., 395

Braud, I., 77

Brewster, J., 395

Buchanan, I.D., 463

Bujoczek, G., 395

Bukhari, A.A., 155

Burke, J.M., S1, S15, S51, S63, S87

Cabrera, F., 335

Calhoun, D.D., 441

Callister, S.J., 247

Carlson, K., 293

Carr, M., 293

Carville, M., 255

Chaala, A., 119

Chanasyk, D.S., S41, S51, S63, S87

Chatellier, P., 429

Chaudhary, D.S., 111

Chen, W., S63, S73

Christoforou, C.S., 441

Clemente, J.S., 177

Cloete, T.E., 273

Cooper, A.T., 407

Coulibaly, H.D., 47

Coy, D.L., 199

Cristiani-Urbina, E., 401

Demarty, J., 77

Desjardins, R., 343

Díaz, M.J., 335

Donnelly, M., S35
Dore, S.Y., 227

Doyon, F., S23

Dudas, M.J., 199, 265

Dueri, S., 213

Duinker, P.N., S23

Ellis, D., 139

Fakhru'l-Razi, A., 299

Fedorak, P.M., 177, 199

Flora, J.R.V., 407

Fogarty, E., 395

Foreman, M.G.G., 163

Foto Menbohan, S., 383

Gabos, S., S63, S73

Gadbois, A., 343

Galíndez-Mayer, J., 401

Gamal El-Din, M., 63, 127, 413

Gao, W., 325

Gauthier, V., 281

Gibbs, S.G., 85

Goel, R.K., 407

Gooding, T., S23

Gregory, D., 293

Grubert, J.P., 99

Gupta, N.K., 453

Haberman, M., 9

Hall, E.R., 17

Hashsham, S.A., 247

Héduit, A., 429

Hirsch, K., S23

Hwang, S., 149

Ikehata, K., 463

Johri, S., 453

Joyce, S., 39

Juárez-Ramírez, C., 401

Kelly, H.G., 187

Kemka, N., 383

Kim, S.H., 111

Koch, F.A., 315, 369, 473

Koudjonou, B., 343

Kulpa, Jr., C.F., 227

Laflamme, E., 139

Larson, M.R., 163

Laurent, P., 237

Laursen, A.E., 227

Lessard, P., 429
Levasseur, B., 355

Levings, C.D., 163

Li, J.Z., 187

Li, B., 27

Liao, P.H., 369

Locat, J., 213

López, R., 335

MacKinnon, M.D., 199

Madejón, E., 335

Maheshwari, M., 453

Mahmood-Khan, Z., 17

Mangeney, A., 77

Mapfumo, E., S51, S87

Mavinic, D.S., 187, 315, 369, 473

Meckes, M.C., 85

Mehrotra, I., 307

Mercier, G., 355

Messouli, M., 383

Meunier, N., 1, 9

Meyer, W.L., S1

Miller, C.A., 83

Millette, R., 281

Millions, D., S63

Min, K.-S., 149

Molla, A.H., 299

Moon, H., 111

Ngo, H.H., 111

Njiné, T., 383

Nola, M., 383

Oleszkiewicz, J., 395

Ottlé, C., 77

Pandey, R., 453

Pineda-Santiago, V., 401

Prepas, E.E., S1, S15, S41, S51, S63, S73, S87

Prévost, M., 237, 343

Putz, G., S1, S63, S87

Rahni, M., 139

Reimers, R.S., 395

Renneberg, A.J., 199

Robinson, H., 255

Rodriguez, M., 139

Rodriguez, M.J., 47

Rodríguez-Mendoza, N., 401

Rothwell, R., S23 
Roy, C., 119

Rudy, A., S23

Ruiz-Ordaz, N., 401

Russell, J.S., S15, S23

Saleem, M., 155

Salmerón-Alcocer, A., 401

Salmon, L.G., 441

Scarpino, P.V., 85

Schauer, J.J., 441

Sego, D.C., 325

Sengupta, B., 453

Serediak, M., S63

Sérodes, J., 139
Servais, P., 237, 383

Sharma, M., 453

Shrivastava, A.K., 307

Shukla, B.P., 453

Simpson, M.J., 199

Smith, D.W., 39, 63, 127, 325, 413, 463, S1, S15, S63, S87

Strachan, W., S73

Stricker, A.-E., 429

Tarbotton, M.R., 163

Tchio, M., 343

Theron, J., 273

Therrien, R., 213
Thottan, J., 233

Tremblay, G., 281

Tyagi, R.D., 1, 9

Van Damme, L., S23

Vigneswaran, S., 111

Walsh, T., 255

Wang, F., 413

Whitson, I., S1

Whitson, I.R., S41, S51

Yen, T.-W., 177

Zahangir Alam, Md., 299

Zébazé Togouet, S.H., 383 